05

\title{
Зависимость коэрцитивной силы от скорости развертки магнитного поля в сплавах (PrDy)(FeCo)B
}

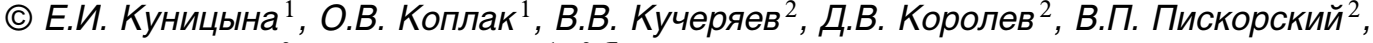 \\ А. Безверхний ${ }^{3}$, Р.Б. Моргунов ${ }^{1-3, \text { व }}$ \\ ${ }^{1}$ Институт проблем химической фризики РАН, \\ Черноголовка, Россия \\ ${ }^{2}$ Всероссийский институт авиационных материалов, \\ Москва, Россия \\ ${ }^{3}$ Тамбовский государственный технический университет, \\ Тамбов, Россия \\ ฯ E-mail: morgunov2005@yandex.ru
}

(Поступила в Редакцию 31 января 2017 г.)

$\mathrm{B}$ спеченных магнитах $\left(\operatorname{Pr}_{1-x} \mathrm{Dy}_{x}\right)_{13}\left(\mathrm{Fe}_{1-y} \mathrm{Co}_{y}\right)_{79} \mathrm{~B}_{8}$ обнаружена зависимость коэрцитивной силы от среднего времени записи петли гистерезиса. Установлено, что в диапазоне средней скорости заметания петли гистерезиса $1.1 \cdot 10^{2}-3 \cdot 10^{5} \mathrm{Oe} / \mathrm{min}$ коэрцитивная сила увеличивается с ее ростом на $22 \%$, подчиняясь логарифмической зависимости от скорости прохождения петли с насыщением при малых скоростях. Методом магнитной силовой микроскопии установлены изменения доменной структуры, вызываемые магнитным полем в процессе намагничивания.

Работа выполнена в рамках реализации комплексного научного направления 11.1 „Термостабильные магнитотвердые материалы и математические модели расчета их температурных характеристик для навигационных приборов нового поколения“ („Стратегические направления развития материалов и технологий их переработки на период до 2030 г.“) и при поддержке гранта 3.1992.2017/ПЧ в рамках конкурса научных проектов, выполняемых научными коллективами исследовательских центров и (или) научных лабораторий образовательных организаций высшего образования.

DOI: $10.21883 /$ FTT.2017.08.44751.24

\section{1. Введение}

Магнитожесткие магнитные сплавы RE-TM-B (RE - редкоземельный металл, ТМ - переходный металл) в настоящее время широко используются в различных устройствах благодаря большой величине их максимального энергетического произведения $(B H)_{\max }$, где $B$ - индукция магнитного поля, пропорциональная остаточной намагниченности $M_{\text {rem }}$, а $H-$ напряженность магнитного поля, пропорциональная коэрцитивной силе $H_{\mathrm{c}}$ [1]. По этой причине усовершенствование постоянных магнитов в основном сводится к попыткам увеличения двух упомянутых величин (коэрцитивной силы и остаточной намагниченности). В технологиях изготовления постоянных магнитов считается значительным успехом увеличение одной из них на $10-20 \%$. В то же время величины $M_{\text {rem }}$ и $H_{C}$ зависят от метода их измерения. В частности, в различных типах магнитов величина коэрцитивной силы зависит от времени прохождения петли магнитного гистерезиса [2-4], которое может варьироваться от нескольких секунд до нескольких часов. Это явление обусловлено конкуренцией между магнитной релаксацией, которая успевает частично протекать во время записи петли гистерезиса, и процессом намагничивания-размагничивания, который определяется динамикой зарождения обратной фазы и скоростью движения доменных стенок. Магнитная релаксация определяется динамикой зарождения фазы обратной намагниченности и временем ожидания открепления доменных стенок от препятствий. Количественной мерой магнитной релаксации является магнитная вязкость $S=d M / d \ln t$, которая характеризует временну́ю зависимость намагниченности $M$ для случая равнораспределения потенциальных барьеров размагничивания по высоте. Магнитная релаксация в ферромагнитных материалах определяет результат измерения магнитного момента и зависит от длительности этого измерения, что редко принимается во внимание и обсуждается в литературе. В частности, релаксация влияет на параметры петли магнитного гистерезиса в зависимости от времени ее заметания, температуры и других условий эксперимента. Магнитный гистерезис записывается различными методами (с помощью эффекта Керра, в импульсном и переменном магнитном поле, на вибро- или СКВИД-магнитометрах и др.). Все эти методы характеризуются различной длительностью прохождения петли гистерезиса в диапазоне от нескольких секунд до нескольких часов. В этом же диапазоне лежат и времена релаксации в сплавах $\mathrm{RE}-\mathrm{TM}-\mathrm{B}$ в зависимости от приложенного магнитного поля и температуры, как показано в наших работах [5-8]. Поэтому можно ожидать, что в магнитожестких магнитах семейства $\mathrm{RE}-\mathrm{TM}-\mathrm{B}$, где магнитная вязкость в значительной степени определяет намагниченность, 
коэрцитивная сила также будет зависеть от скорости заметания магнитного поля при получении петли магнитного гистерезиса. Цель настоящей работы заключается в установлении степени чувствительности коэрцитивной силы к скорости развертки магнитного поля при прохождении петли гистерезиса в спеченных магнитах $\left(\operatorname{Pr}_{1-x} \mathrm{Dy}_{x}\right)_{13}\left(\mathrm{Fe}_{1-y} \mathrm{Co}_{y}\right)_{79} \mathrm{~B}_{8}$, а также в установлении взаимосвязи этих параметров с величиной магнитной вязкости и изменением доменной структуры магнитов.

\section{2. Методика}

Образцы $\left(\operatorname{Pr}_{1-x} \mathrm{Dy}_{x}\right)_{13}\left(\mathrm{Fe}_{1-y} \mathrm{Co}_{y}\right)_{79} \mathrm{~B}_{8}$ были получены путем спекания частиц размером 500-600 $\mu \mathrm{m}$ в высокотемпературной вакуумной печи. Методика получения, а также химический и фазовый анализ подробно описаны в работе [9]. Параметры тетрагональной ячейки основной магнитной фазы близки к параметрам сплава $\mathrm{Nd}_{2} \mathrm{Fe}_{14} \mathrm{~B}$ (2-14-1), для которого $a=8.80 \AA, c=12.20 \AA$ [2]. Объем элементарной ячейки $V_{0}=944.768 \AA^{3}$, общее число элементарных ячеек в объеме образца составляет $N_{C}=V / V_{0} \approx 2.8 \cdot 10^{18}$.

Для образцов, имеющих форму пластинки размером $0.5 \times 1.5 \times 3.5 \mathrm{~mm}$, были выполнены измерения магнитного момента в интервале магнитных полей от -50 до $+50 \mathrm{kOe}$. Изображения доменной структуры в предварительно намагниченных образцах были получены с помощью атомного силового микроскопа Aist-NTSmartSPM (MFM) в отсутствие магнитного поля. Измеряемые значения магнитной силы, пропорциональные градиенту магнитного поля вблизи поверхности образца, были также пропорциональны частотному сдвигу вибрирующего кантилевера на основе $\mathrm{CoCr}$ с коэрцитивной силой $400 \mathrm{Oe}$ в режиме „tapping-lift“. Силовая константа и резонансная частота кантилевера были равны $5 \mathrm{~N} / \mathrm{m}$ и $196 \mathrm{kHz}$ соответственно. Расстояние кантилевера от поверхности составляло $80 \mathrm{~nm}$ и было одинаковым во всех опытах.

Все измерения магнитного момента образцов проводились при температуре $T=300 \mathrm{~K}$, в процессе измерения температура образца поддерживалась с точностью $0.1 \mathrm{~K}$. Внешнее магнитное поле было направлено вдоль легкой оси намагничивания образца. Намагниченность в постоянном магнитном поле $M$ была измерена двумя способами с разной скоростью развертки магнитного поля: в области скоростей заметания $v=1.1 \cdot 10^{2}-3 \cdot 10^{3} \mathrm{Oe} / \mathrm{min}$ с помощью магнитометра MPMS 5XL Quantum design. В области скоростей заметания $v=3 \cdot 10^{3}-3 \cdot 10^{5} \mathrm{Oe} / \mathrm{min}$, с помощью гистерезисографа Permagraph C-300. В первом случае вариация средней скорости заметания достигалась за счет сокращения числа точек при измерении петли гистерезиса образца в СКВИД-магнитометре, во втором - путем увеличения скорости изменения подаваемого тока в соленоиде гистерезисографа.

\section{3. Экспериментальные результаты и обсуждение}

Доменная структура образца была исследована как в режиме атомно-силовой микроскопии (ACM), так и в режиме сканирования градиента магнитного поля вблизи поверхности образца - магнитно-силовой микроскопии (МСМ) (рис. 1). На рис. 1 результаты ACM, содержащие царапины, не показаны. Образец, используемый в этих опытах, имел тонко шлифованную поверхность и позволял поддерживать удаление кончика кантилевера от поверхности на уровне $100 \mathrm{~nm}$. На рис. 1 представлены МСМ-сканы поверхности до и после воздействия на нее магнитного поля в течение различного времени. Рельефы АСМ и МСМ были различны, что свидетельствует о том, что магнитное изображение обусловлено не перепадами высоты микрорельефа, а отражает распределение намагниченности доменной структуры и зерен. На фотографиях видны полосовые домены со средним линейным размером $\sim 10 \mu \mathrm{m}$. Домены расположены внутри зерен, которые отчетливо различаются в виде округлых областей со средним размером $40 \times 40 \mu \mathrm{m}$. Аналогичные по конфигурации полосовые домены были ранее обнаружены в образцах семейства $\mathrm{Nd}-\mathrm{Fe}-\mathrm{B}$ в [10].

Для определения роли магнитного поля в формировании доменной структуры к исходному (не намагниченному преднамеренно) образцу после первого контрольного измерения, выявляющего первоначальную доменную структуру (рис. $1, a$ ), подносили постоянный магнит, обеспечивавший в месте расположения образца магнитное поле $\sim 100$ Ое. После выдержки образца в таком магнитном поле его положение в микроскопе сохранялось, так что последующий скан (после удаления магнита) получался для того же участка поверхности (рис. $1, b)$. Из рис. $1, b$ видно, что после первой экспозиции в магнитном поле в течение $3 \mathrm{~min}$ произошло перераспределение доменов, расположение доменов изменялось и при последующем поднесении магнита, обеспечившем длительность экспозиции $9 \mathrm{~min}$ (рис. 1, $c$ ). В нашей работе не было возможности измерить изменения распределения намагниченности in situ в магнитном поле, однако статичные изображения, изменяющиеся при каждом намагничивании, указывают на зависимость процесса намагничивания от времени экспозиции в магнитном поле в минутной временно́й шкале. Можно сделать вывод, что за магнитную релаксацию ответственны домены, а не зародыши обратной намагниченности, что соответствует результатам работы [5], полученным другими косвенными методами.

На рис. 2 представлены фрагменты петель гистерезиса образца, полученные на СКВИД-магнитометре при различной скорости развертки магнитного поля. С увеличением скорости развертки магнитного поля увеличивается значение коэрцитивной силы, подобный эффект наблюдался в [3]. Поскольку СКВИД-магнитометр не позволяет записывать петлю гистерезиса со скоростью, 
большей $3 \cdot 10^{3} \mathrm{Oe} / \mathrm{min}$, экспериментальные точки для бо́льших скоростей были получены с помощью гистерезисографа. Обобщенная зависимость коэрцитивной
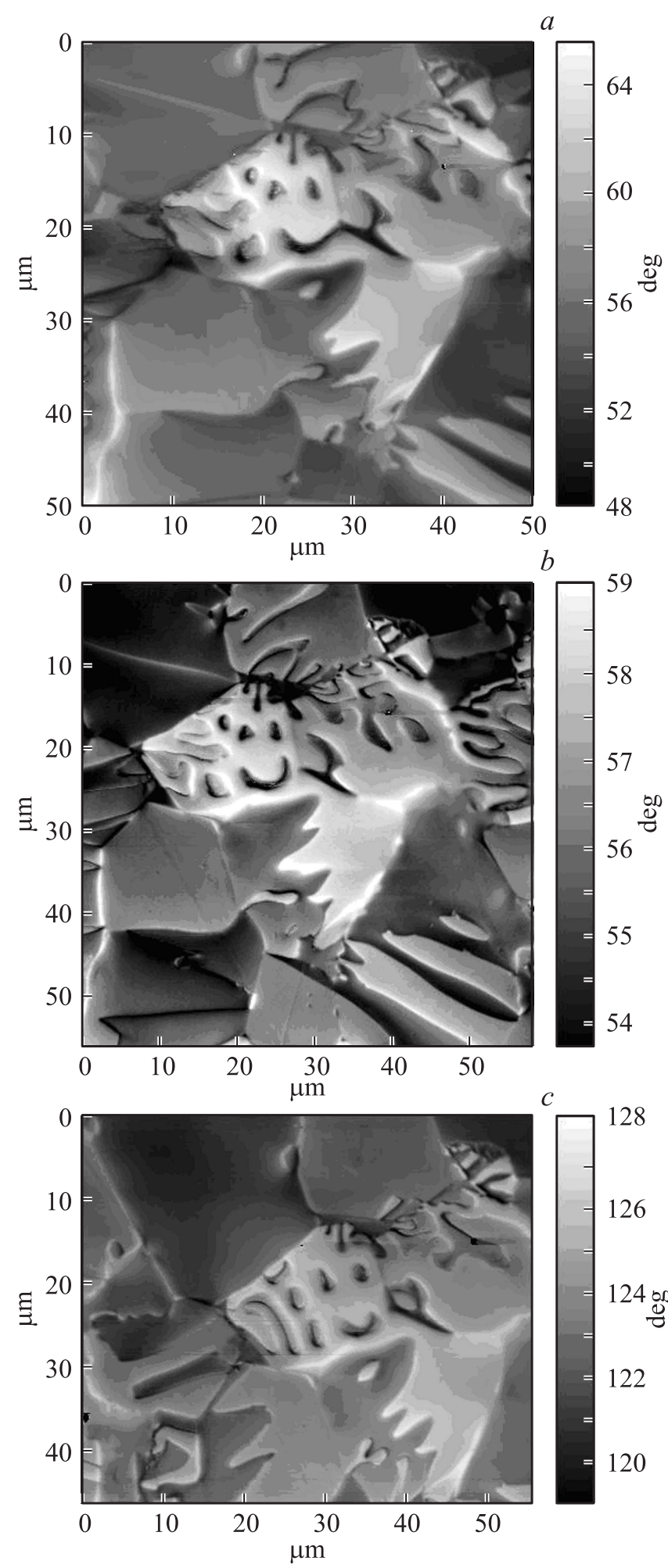

Рис. 1. Распределение вертикального градиента магнитного поля, создаваемого образцом вблизи его поверхности. $a-$ исходное состояние образца; $b$ и $c$ - после воздействия магнитного поля в течение 3 и 9 min соответственно.

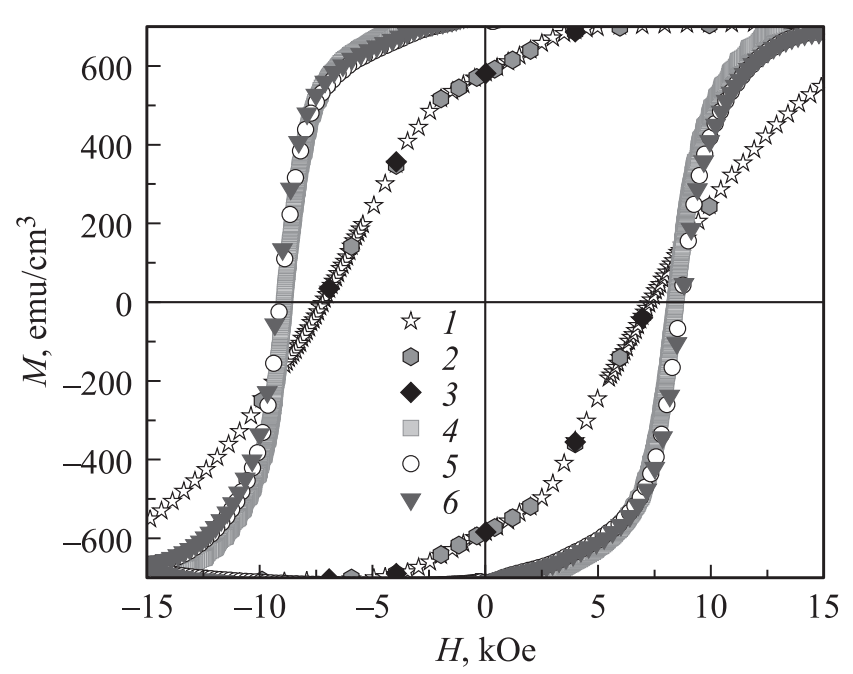

Рис. 2. Фрагменты петель магнитного гистерезиса образца, записанные со скоростью развертки поля 0.3 (1), 1.8 (2), $3.8(3), 19.5(4), 88.7$ (5) и $213 \mathrm{kOe} / \mathrm{min}(6)$.

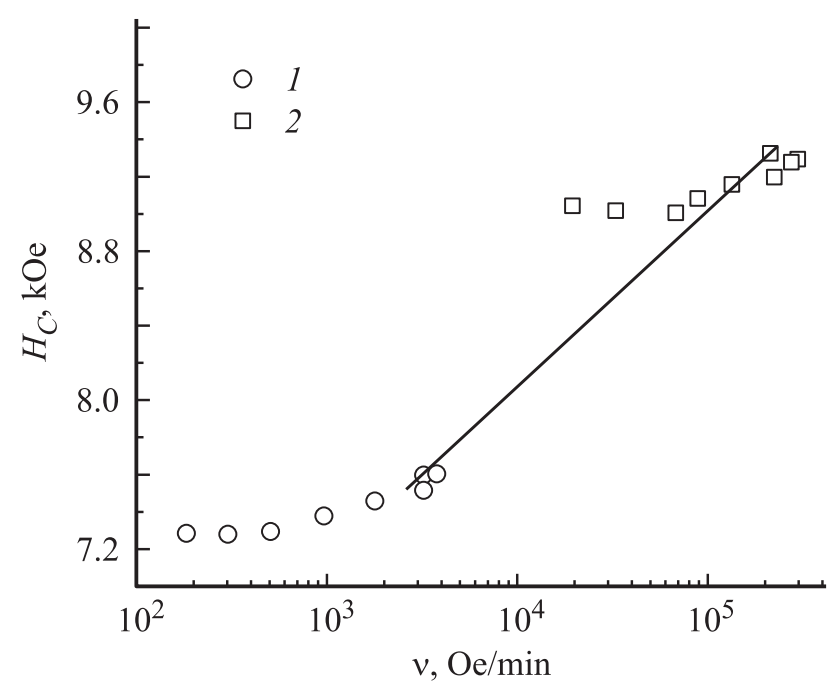

Рис. 3. Зависимость коэрцитивной силы образца от скорости развертки поля. 1 - точки, полученные с помощью СКВИД-магнитометра, 2 - точки, полученные с помощью гистерезисографа. Сплошной линией показана аппроксимация логарифмической зависимостью.

силы от скорости развертки магнитного поля представлена на рис. 3. В полулогарифмических координатах зависимость $H_{C}(v)$ при $v>5 \cdot 10^{2} \mathrm{Oe} / \mathrm{min}$ может быть аппроксимирована логарифмической зависимостью для определения магнитной вязкости. При этом используют так называемую приведенную вязкость $S_{v}$, не зависящую от формы образца [3],

$$
S_{v}=S\left(1-N \chi_{\mathrm{rev}}\right) / \chi_{\mathrm{irr}}
$$

где $\chi_{\text {rev }}$ и $\chi_{\text {irr }}-$ необратимая и обратимая части магнитной восприимчивости, $N-$ размагничивающий фактор плоской пластинки. 
С учетом полученных ранее значений $\chi_{\mathrm{rev}}, \chi_{\text {irr }}$ и $N[8]$ приведенная вязкость была определена нами из наклона кривой $H_{C}(\lg v)$ (рис. 3) в соответствии с формулой [3]

$$
S_{v}=\left(H_{C 1}-H_{C 2}\right) / \ln \left[\left(d H_{1} / d t\right) /\left(d H_{2} / d t\right)\right],
$$

где $H_{C 1}$ и $H_{C 2}$ - значения коэрцитивной силы, полученные при скоростях заметания магнитного поля $v_{1}=d H_{1} / d t$ и $v_{2}=d H_{2} / d t$ соответственно.

В целом же зависимость $H_{C}(v)$ имеет немонотонный характер, претерпевая насыщение средней скорости заметания поля $v<5 \cdot 10^{2} \mathrm{Oe} / \mathrm{min}$. Ниже этого значения скорости заметания коэрцитивная сила перестает зависеть от скорости изменения магнитного поля, и устанавливается квазистатический режим намагничивания, при котором доменная структура „успевает“ подстраиваться под новое значение магнитного поля в каждой точке петли магнитного гистерезиса.

Для участка зависимости $H_{C}(v)$, полученного на СКВИД-магнитометре, приведенная магнитная вязкость составила $\mu_{0} S_{v}=2.1 \mathrm{mT}$, для участка, записанного на гистерезисографе, $\mu_{0} S_{v}=3.5 \mathrm{mT}$, а среднее значение, определенное для аппроксимации всей зависимости $H_{C}(v)$ при $v>5 \cdot 10^{2} \mathrm{Oe} / \mathrm{min}$, составило $2.8 \mathrm{mT}$. Полученные значения $\mu_{0} S_{v}$ близки к величинам магнитной вязкости для наночастиц $\mathrm{BaO}_{6} \mathrm{Fe}_{2} \mathrm{O}_{3}$ в поликристаллах $\mathrm{SmCo}_{3} \mathrm{Cu}_{2}$ [3] и хорошо согласуются с вязкостью, определенной нами ранее для образцов $(\mathrm{SmNdDy})(\mathrm{FeCo}) \mathrm{B}$ при $300 \mathrm{~K}$ в обратном магнитном поле $10 \mathrm{kOe}$ [8]. Отметим, что в [8] значение магнитной вязкости было получено из временно́й зависимости магнитного момента, т. е. независимым методом.

\section{4. Заключение}

$\mathrm{B}$ спеченных магнитах $\left(\operatorname{Pr}_{1-x} \mathrm{Dy}_{x}\right)_{13}\left(\mathrm{Fe}_{1-y} \mathrm{Co}_{y}\right)_{79} \mathrm{~B}_{8}$ наблюдается уменьшение коэрцитивной силы с ростом средней скорости заметания магнитного поля при записи петли магнитного гистерезиса. Расчет магнитной вязкости из зависимости коэрцитивной силы от скорости заметания приводит к величине магнитной вязкости, близкой к значениям, полученным другими независимыми способами. Это свидетельствует о том, что магнитная релаксация в процессе записи гистерезиса является главной причиной чувствительности коэрцитивной силы к скорости изменения магнитного поля. Магнитная силовая микроскопия свидетельствует о зависимости доменной структуры от времени экспозиции образца в магнитном поле в минутной шкале при $\sim 100 \mathrm{Oe}$, что указывает на то, что при $300 \mathrm{~K}$ магнитная вязкость определяется движением доменов в образцах.

\section{Список литературы}

[1] J.F. Herbst. Rev. Mod. Phys. 63, 819 (1991).

[2] S.J. Collocott, J.B. Dunlop. J. Magn. Magn. Mater. 320, 2089 (2008).
[3] R. Grössinger, R. Sato Turtelli, C. Téllez-Blanco. J. Optoelectron. Adv. Mater. 6, 557 (2004).

[4] X. Xu, T. Kihara, M. Tokunaga, A. Matsuo, W. Ito, R.Y. Umetsu, K. Kindo, R. Kainuma. Appl. Phys. Lett. 103, 122406 (2013).

[5] E.N. Kablov, O.G. Ospennikova, D.E. Kablov, V.P. Piskorskii, E.I. Kunitsyna, A.I. Dmitriev, R.A. Valeev, D.V. Korolev, I.I. Rezchikova, A.D. Talantsev, R.B. Morgunov. J. Appl. Phys. 117, 243903 (2015).

[6] E. Kablov, O. Ospennikova, V. Piskorskii, D. Korolev, A. Dmitriev, E. Kunitsyna, R. Morgunov. Eur. Phys. J. Plus 131, 40 (2016).

[7] R.B. Morgunov, E.I. Kunitsyna, V.V. Kucheryaev, V.P. Piskorskii, O.G. Ospennikova, E.N. Kablov. Eur. Phys. J. Plus 131, 344 (2016).

[8] А.И. Дмитриев, А.Д. Таланцев, Е.И. Куницына, Р.Б. Моргунов, В.П. Пискорский, О.Г. Оспенникова, Е.Н. Каблов. ЖЭТФ 150, 350 (2016).

[9] Е.Н. Каблов, О.Г. Оспенникова, В.П. Пискорский, И.И. Резчикова, Р.А. Валеев, Е.А. Давыдова. Авиационные материалы и технологии $S 2,5$ (2015).

[10] Er. Girt, K.M. Krishnan, G. Thomas, E. Girt, Z. Altounian. J. Magn. Magn. Mater. 231, 219 (2001). 